\title{
Growth, HRXRD, Microhardness and Dielectric Studies on the NLO Material L-Alaninium Maleate
}

\author{
S.A. Martin Britto Dhas ${ }^{1}$, G. Bhagavannarayana ${ }^{2}$ and S. Natarajan*,1 \\ ${ }^{1}$ Department of Physics, Madurai Kamaraj University, Madurai - 625 021, India \\ ${ }^{2}$ Material Characterization Division, National Physical Laboratory, New Delhi-110012, India
}

\begin{abstract}
The single crystals of the NLO material, L-Alaninium maleate were grown by using the submerged seed solution method. The identity of the crystal was confirmed by single crystal X-ray diffraction. The crystalline perfection was analyzed using high resolution X-ray diffraction and it was found that the crystalline perfection is quite good. The values of the laser damage threshold and the Vicker's microhardness are in the higher range. The dielectric studies at different temperatures showed that the dielectric constant and dielectric loss have low values at higher frequencies and these values are independent of the temperature. The details are presented and discussed.
\end{abstract}

Keyword: X-ray diffraction, Growth from solution, Organic compound, Nonlinear optical materials.

\section{INTRODUCTION}

Nonlinear optical processes provide the key functions of frequency conversion and optical switching. These applications depend upon the various properties of the materials, such as transparency, birefringence, laser damage threshold, hardness, dielectric constant, thermal, photochemical and chemical stability. A distinct advantage of organic electrooptic materials over the inorganic electro-optic is their exceptional bandwidth potential. Both the index of refraction and dielectric permittivity are determined by the $\pi$ electrons and both properties are relatively independent of frequency. This means that optical and long wavelength (radiowave, microwave and millimetre wave) fields propagate at the same velocity, permitting long interaction lengths to be exploited. The high dielectric constants of inorganic materials such as lithium niobate lead to power dissipation, providing yet another advantage for the low dielectric constant organic materials [1]. L-Alaninium maleate (LAM) is one of the recently developed organic NLO crystals from our laboratory. The details regarding the growth, spectroscopic, second harmonic generation and thermal properties were reported elsewhere [2,3]. In this article, details regarding the crystalline perfection, laser damage threshold, microhardness and dielectric constants and loss at different temperatures are reported.

\section{EXPERIMENTAL STUDIES}

\section{Crystal Growth}

L-Alanine and maleic acid (E. Merck (India) Ltd.) were mixed in the stoichiometric ratio of 1:1 in distilled water and the solution was allowed to evaporate at room temperature. Small well-defined, transparent crystals were obtained in a few days. Some good quality crystals were selected as seeds

*Address correspondence to this author at the Department of Physics, Madurai Kamaraj University, Madurai - 625 021, India; Tel: 91-04522458471- 352 (extn); Fax: 91-452-2459181;

E-mail: s_natarajan50@yahoo.com and the remaining crystals were powdered and dissolved in distilled water to obtain a saturated solution. This solution was filtered using a micro filter paper. A suitable seed crystal was selected and kept at the bottom of the vessel containing the saturated solution and optimally closed using a perforated polythene sheet. Transparent bulk crystal of size: 19.0 $\times 6.0 \times 5.0 \mathrm{~mm}^{3}$ (Fig. 1) crystallized within two weeks.

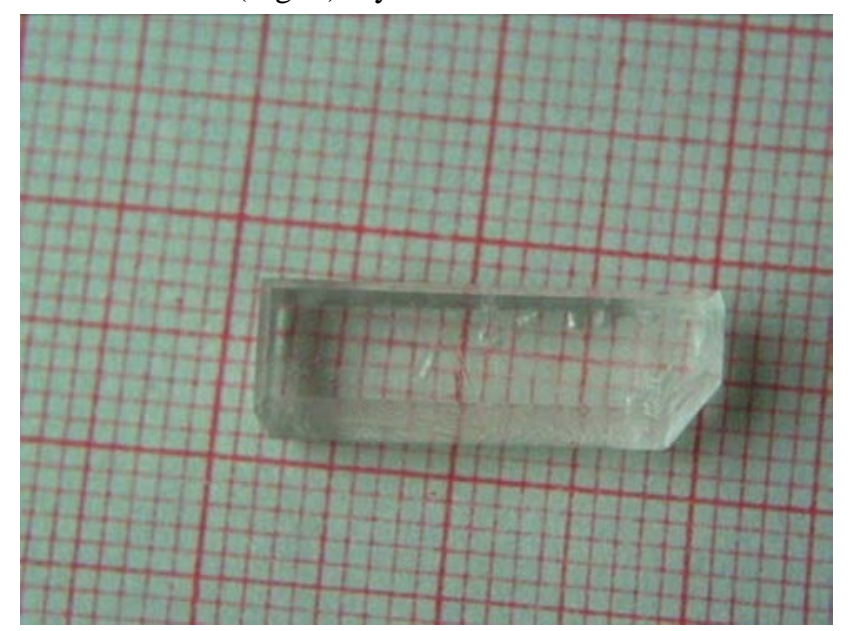

Fig. (1). Photograph of LAM crystal.

\section{Characterization}

Nonius CAD-4/MACH 3 diffractometer with MoKa $(0.71073 \AA)$ radiation was used to obtain the accurate cell parameters of the grown crystals at room temperature, by the least-squares refinement of the setting angles of 25 reflections. The optical and structural homogeneity of NLO crystals have paramount importance for any optical device. The recent work [4] shows that the HRXRD is a very good tool to investigate the crystalline perfection of NLO crystals. To reveal the crystalline perfection of the grown crystals, a multi crystal X-ray diffractometer (MCD) developed at the National Physical Laboratory (NPL), New Delhi [5] was 
used to record the high-resolution diffraction curve (DC) for (200) diffracting plane in symmetrical Bragg geometry. Before recording the diffraction curve, to remove the noncrystallized solute atoms remaining on the surface of the crystal and also to ensure the surface planarity, the specimen was first lapped and chemically etched in a non preferential etchant of water and acetone mixture in 1:2 volume ratio. The nonlinear optical conversion efficiencies were tested using a modified setup of Kurtz and Perry [6]. A Q-switched $\mathrm{Nd}$ :YAG laser beam of wavelength $1064 \mathrm{~nm}$ was used with an input power of $2.0 \mathrm{~mJ}$ and pulse width of $10 \mathrm{~ns}$, the repetition rate being $10 \mathrm{~Hz}$. The crystals were ground to a uniform particle size of about $125-150 \mu \mathrm{m}$ and then packed in a capillary of uniform bore and exposed to laser radiations. The second harmonic signal generated in the crystalline sample was confirmed from the emission of green radiation $(\lambda=532 \mathrm{~nm})$ from the crystal. The intensity of the green light was measured using a photomultiplier tube. The laser damage threshold studies on LAM have been carried out using a Q-switched Nd:YAG laser of $10 \mathrm{~ns}$ pulses at a wavelength of $1064 \mathrm{~nm}$. The energy density was calculated using the formula,

$$
\text { Energy density }=\mathrm{E} / \mathrm{A}\left(\mathrm{GW} / \mathrm{cm}^{2}\right) \text {, }
$$

where $\mathrm{E}$ is the input energy measured in milli-joules and $\mathrm{A}$, the area of the circular spot. The microhardness of the grown crystals was measured using a Shimadzu Microhardness Tester (Model No HMV2T) with diamond indenter. The well-polished crystal was mounted on the platform of the microhardness tester and loads of different magnitudes (10, $25 \mathrm{gm}$ ) were applied over a fixed interval of time. The indentation time was fixed as $15 \mathrm{~s}$. The dielectric study on the crystal was carried out using the instrument, HIOKI 352-50 LCR HITESTER. The measurement was carried out at different temperatures viz., $35^{\circ} \mathrm{C}, 55^{\circ} \mathrm{C}, 75^{\circ} \mathrm{C}$ and $95{ }^{\circ} \mathrm{C}$. A sample of LAM of dimensions: $3.47 \times 3.00 \times 2.27 \mathrm{~mm}^{3}$ was used for the measurements. Silver coating was applied on the opposite sides of the crystal, which was placed between two copper electrodes and thus a parallel plate capacitor was formed. The capacitance of the sample was measured for various frequencies, in the range: $500 \mathrm{~Hz}$ to $5 \mathrm{MHz}$. The dielectric constant was calculated by using the relation:

$$
\varepsilon^{\prime}=\mathrm{Ct} /\left(\varepsilon_{\mathrm{o}} \mathrm{A}\right)
$$

where $C$ is the capacitance, $t$, the thickness of the sample, $\varepsilon_{o}$, the permittivity of free space and A, the area of cross section.

\section{RESULTS AND DISCUSSION}

\section{Single Crystal X-Ray Diffraction}

LAM crystallized in the monoclinic system and the lattice parameters were found to be: $a=5.588(2) \AA, b=$ 7.380 (4) $\AA, c=23.699$ (1) $\AA$ and the space group is $\mathrm{P} 2{ }_{1} 2_{1} 2_{1}$. These values agreed well with the reported values [7].

\section{High-Resolution X-Ray Diffraction (HRXRD) Analysis}

As seen in Fig. (2), the DC is quite sharp without any satellite peaks which may otherwise be observed either due to internal structural grain boundaries [8] or due to epitaxial layer which may some times form, for example in case of complexing layers formed due to organic additives [9]. The full width at half maximum (FWHM) of the diffraction curve is 36 arc sec. Though this value is not very close to that expected for an ideally perfect crystal $[0.42$ arc $s$ for the present specimen for (200) diffracting planes using MoK $\alpha_{1}$ radiation] from the plane wave theory of dynamical X-ray diffraction [10], it is close to that expected for a nearly perfect real life crystal. The single sharp diffraction curve with very low FWHM indicates that the crystalline perfection is quite good.

The specimen is a nearly perfect single crystal without any internal structural grain boundaries and dislocations (or very low density of dislocations which could not be detected by high-resolution X-ray topography).

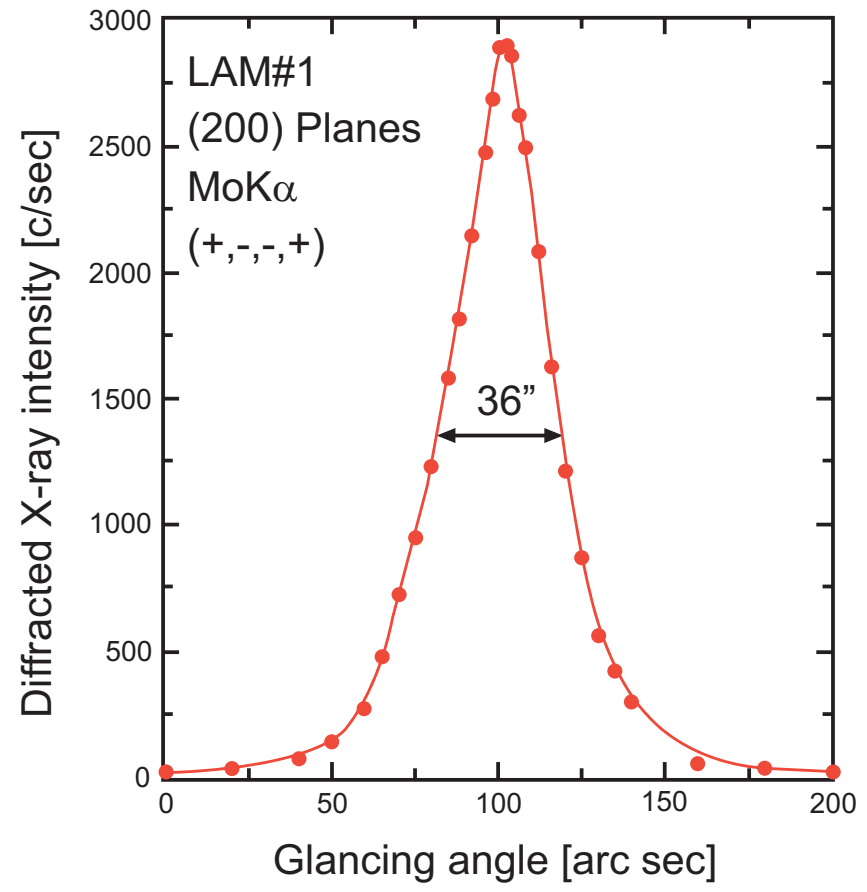

Fig. (2). High-resolution X-ray diffraction curve recorded for LAM single crystal in symmetrical Bragg geometry for (200) diffracting planes using $\mathrm{MoK} \alpha_{1}$ radiation.

\section{SECOND H ARMONIC GENERATION AND LASER DAMAGE THRESHOLD STUDIES}

The second harmonic signal of $55 \mathrm{mV} /$ pulse for LAM was obtained for an input energy of $2.0 \mathrm{~mJ} / \mathrm{pulse}$. But the standard KDP crystal gave a SHG signal of $45 \mathrm{mV} /$ pulse for the same input energy. One of the decisive criteria for a NLO crystal to perform as a device is its resistance to laser damage, since high optical intensities are involved in nonlinear processes. Inorganic crystals are usually known to have high resistance to laser damage. The laser damage energy density was found to be $4.9 \mathrm{GW} / \mathrm{cm}^{2}$. This value is in the higher range compared to some of the recently reported organic NLO materials (Table 1).

\section{Microhardness Studies}

A plot between the load and the hardness number (for the plane 200 ) is shown in Fig. (3), which shows that the microhardness number increases with increasing load. On further increase of the load beyond $50 \mathrm{~g}$, the Hv value started to decrease, indicating the starting of fracture on the surface of the crystal. 
Table 1. The Values of SHG and the Laser Damage Threshold for Some Organic, Semiorganic and Inorganic Materials

\begin{tabular}{|c|c|c|}
\hline Compound & SHG Signal (mV) & ${\left.\text { Laser Damage Threshold (GW/cm }{ }^{2}\right)}$ \\
\hline \hline Potassium dihydrogen phosphate(KDP) & 45 & $0.2^{\mathrm{a}}$ \\
\hline Urea & 270 & $2.5^{\mathrm{a}}$ \\
\hline Benzimidazole & 202 & $2.9^{\mathrm{a}}$ \\
\hline p-Barium borate & 180 & $5.0^{\mathrm{a}}$ \\
\hline L-Arginine phosphate & 135 & $10.0^{\mathrm{a}}$ \\
\hline L-Prolinium tartrate & 43 & $5.9^{\mathrm{b}}$ \\
\hline L-Tartaric acid & 40 & $5.4^{\mathrm{c}}$ \\
\hline L-Alaninium maleate & 55 & $4.9^{\mathrm{d}}$ \\
\hline
\end{tabular}

${ }^{\mathrm{a}}$ Ref. [11], ${ }^{\mathrm{b}}$ Ref. [12], ${ }^{\mathrm{c}}$ Ref. [13], ${ }^{\mathrm{d}}$ Present work.

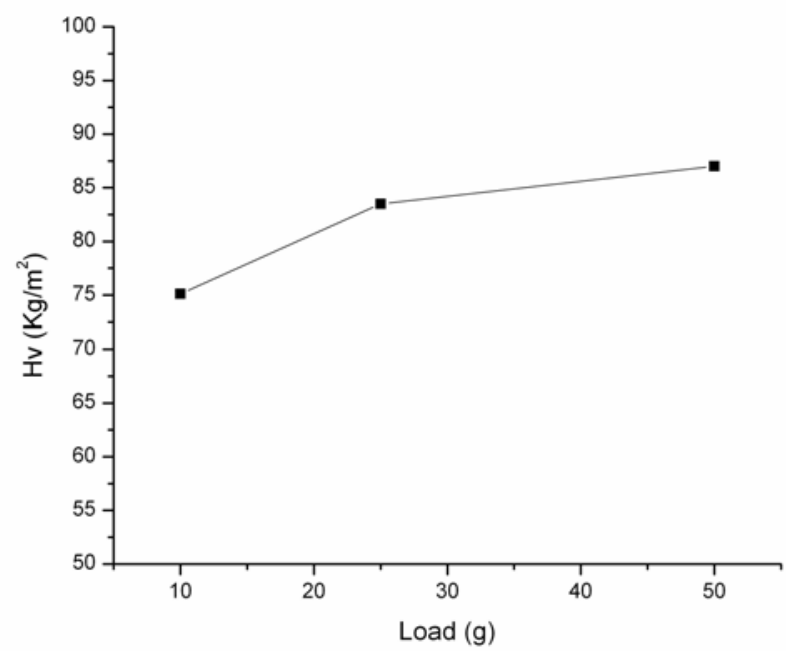

Fig. (3). Plot of microhardness against load.

\section{Dielectric Studies}

An important property of a dielectric is its ability to support an electrostatic field while dissipating minimal energy in the form of heat. The lower the dielectric loss (the proportion of energy lost as heat), the more effective is a dielectric material and the crystals with high dielectric constant lead to power dissipation. Figs. (4 and 5) show that the dielectric constant and the dielectric loss are both inversely proportional to frequency. This is a normal dielectric behaviour [14] and both the dielectric constant $\left(\varepsilon^{\prime}\right)$ and the dielectric loss (D) decrease with increasing frequency (Figs. 4 and $\mathbf{5}$ ). This can be understood on the basis that the mechanism of polarization is similar to that of the conduction process. The electronic exchange of a number of ions in the crystals gives local displacement of electrons in the direction of the applied field, which in turn gives rise to polarization. As the frequency increases, a point will be reached where the space charge cannot sustain and comply with the external field and hence the polarization decreases, giving rise to diminishing values of $\varepsilon^{\prime}$ and $\mathrm{D}$. Continuous gradual decrease in $\mathrm{D}$ as well as $\varepsilon^{\prime}$ suggests that LAM crystal, like any normal dielectric, may have domains of different sizes and varying relaxation times. The high value of $\varepsilon$ ' at lower frequencies may be due to the presence of all the four polarizations, namely space charge, orientational, electronic and ionic polarization and its low value at higher frequencies may be due to the loss of significance of these polarizations gradually. In Fig. (4), a peak at around $800 \mathrm{~Hz}$ at the temperature of $55^{\circ} \mathrm{C}$, may be correlated to the resonance effects on electro-optic coefficients, at certain frequencies. The reason for this may be that for certain critical frequencies of the applied field, standing waves are established in the crystal through the inverse piezoelectric effect and these critical frequencies are dependent on the dimensions of the sample studied. The resonance of these stationary waves with the applied electric field at selected frequencies leads to the observed peaks [15].

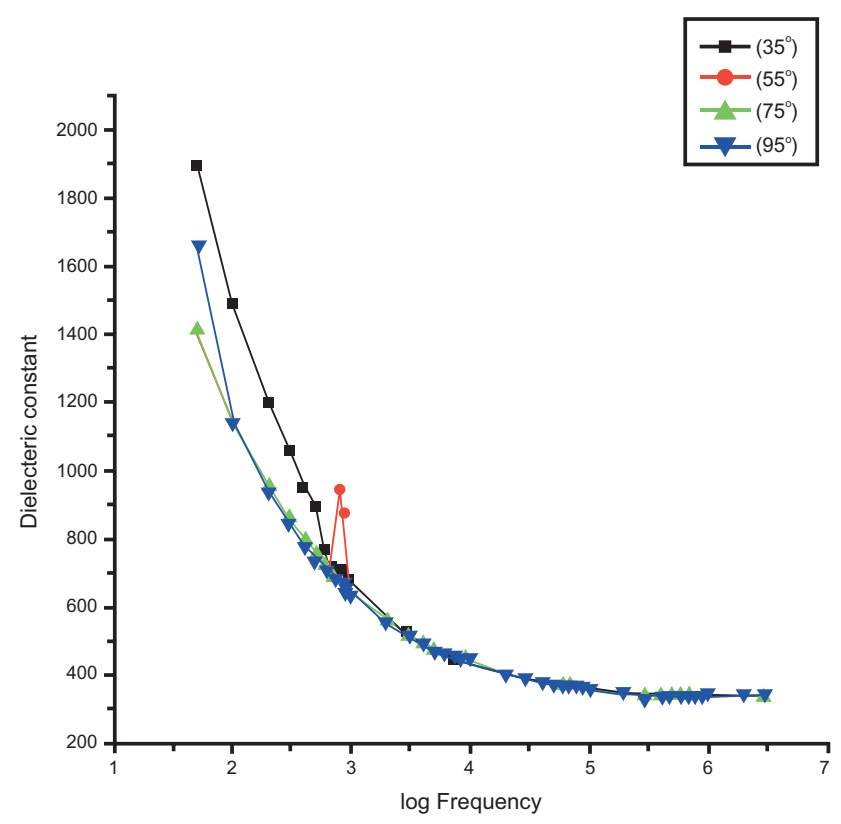

Fig. (4). Variation of dielectric constant of LAM with log frequency.

\section{CONCLUSIONS}

Good quality single crystals of the NLO material LAlaninium maleate was grown by using the submerged-seed 


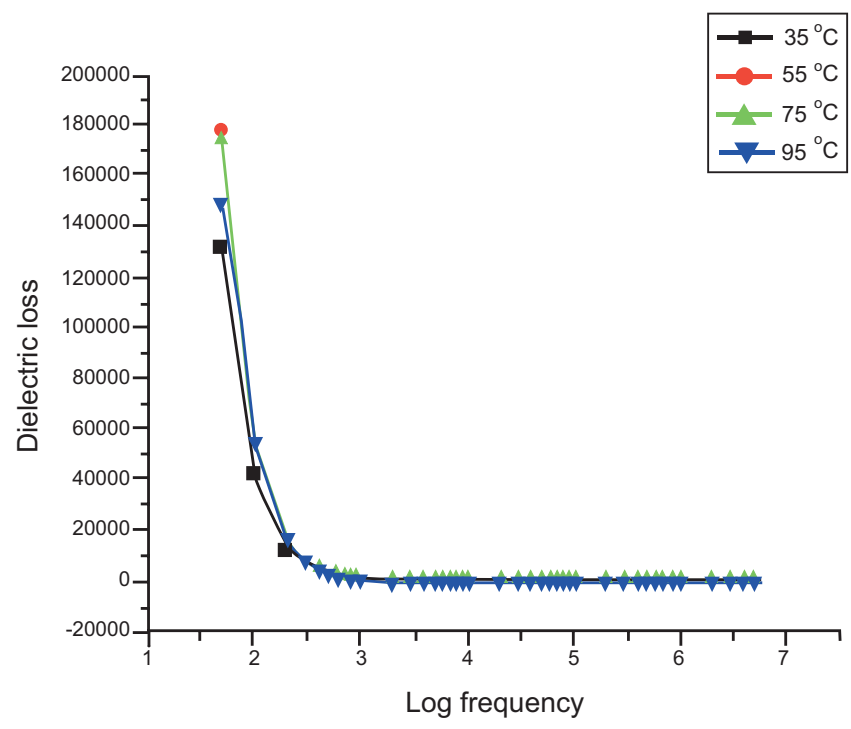

Fig. (5). Variation of dielectric loss of LAM with log frequency.

solution method. High resolution X-ray diffraction showed that the crystalline perfection is quite good. The crystal has high laser damage threshold and it is a hard material. The dielectric constant and dielectric loss of the crystal were studied for a wide range of frequencies at different temperatures. The dielectric loss is almost zero for a wide range of frequencies and it is independent of temperature. The above results showed that the crystal may have some useful applications in nonlinear optics.

\section{ACKNOWLEDGEMENTS}

The authors thank the DST-FIST and the UGC-DRS Programmes and SAMB thanks the Madurai Kamaraj University for providing a Research Fellowship.

\section{REFERENCES}

Dalton LR. Matter, Rational design of organic electro-optic materials. J Phys Condens Matter 2003; 15: R897-R934.
[2] Natarajan S, Martin Britto SA and Ramachandran E. Growth, thermal, spectroscopic, and optical studies of L-alaninium maleate, a new organic nonlinear optical material. Cryst Growth Des 2006; 6: $137-140$.

[3] Martin Britto Dhas SA, Ramachandran E, Raji P, Ramachandran K and Natarajan S. Photoacoustic studies on the thermal properties of the NLO compound: L-alaninium maleate. Cryst Res Technol 2007; 42: 601-606.

[4] Kar S, Ryu H and Bartwal KS. High Resolution XRD Studies on $\mathrm{Mg}, \mathrm{Cr}$ and Nd Doped $\mathrm{LiNbO}_{3}$ Crystals. Open Crystallogr J 2008; 1: $1-5$.

[5] Krishan Lal and Bhagavannarayana G. A high-resolution diffuse $\mathrm{X}$-ray scattering study of defects in dislocation-free silicon crystals grown by the float-zone method and comparison with Czochralskigrown crystals. J Appl Cryst 1989; 22: 209-215.

[6] Kurtz SK and Perry TT. A powder technique for the evaluation of nonlinear optical materials. J Appl Phys 1968; 39: 3798 -3813.

[7] Alagar M, Krishnakumar RV, Subha Nandhini M and Natarajan S. L-Alaninium maleate. Acta Cryst 2001; E57: 0855-0857.

[8] Bhagavannarayana G, Ananthamurthy RV, Budakoti GC, Kumar B and Bartwal KS. A study of the effect of annealing on Fe-doped LiNbO3 by HRXRD, RT and FT-IR. J Appl Cryst 2005; 38: 768771.

[9] Bhagavannarayana G, Parthiban S and Meenakshisundaram S. Enhancement of crystalline perfection by organic dopants in ZTS, ADP and KHP crystals as investigated by high-resolution XRD and SEM. J Appl Cryst 2006; 39: 784-790.

[10] Betterman BW and Cole H. Dynamical Diffraction of X Rays by Perfect Crystals. Rev Mod Phys 1964; 36: 681-717.

[11] Vijayan N, Bhagavannarayana G, Ramesh KR, Gopalakrisnan R, Maurya KK and Ramasamy P. A comparative study on solutionand bridgman-grown single crystals of benzimidazole by highresolution X-ray diffractometry, fourier transform infrared, microhardness, laser damage threshold, and second-harmonic generation measurements. Cryst Growth Des 2006; 6: 1542-1546.

[12] Martin Britto Dhas SA and Natarajan S. Growth and characterization of L-prolinium tartrate - A new organic NLO material. Cryst Res Technol 2007; 42: 471-476.

[13] Martin Britto Dhas SA, Bhagavanarayana G and Natarajan S. Growth and characterization of L-tartaric acid, an NLO material. J Cryst Growth 2007; 309: 48 -52.

[14] Anderson JC. Dielectrics. Chapman and Hall, 1965.

[15] Brahadeeswaran S, Bhat HL, Kini NS, Umarji AM, Balaya P and Goyal PS. Dielectric, thermal, and mechanical properties of the semiorganic nonlinear optical crystal sodium p-nitrophenolate dihydrate. J Appl Phys 2000; 88: 5935-5940.

(C) Dhas et al.; Licensee Bentham Open.

This is an open access article licensed under the terms of the Creative Commons Attribution Non-Commercial License (http://creativecommons.org/licenses/by-nc/3.0/) which permits unrestricted, non-commercial use, distribution and reproduction in any medium, provided the work is properly cited. 